\title{
CHARACTERIZATIONS OF GENERALIZED UNISERIAL ALGEBRAS $\left({ }^{1}\right)$
}

\author{
BY \\ DRURY W. WALL
}

1. Introduction. Let $A$ be a finite dimensional algebra with unit element over a field $K$. In recent years a number of classes of algebras in which the radical may not be zero have been studied. One of the main classifications has been the Frobenius type algebras which can be defined in terms of dualities between left and right ideals of the algebra. Another main group has been the uniserial type algebras which can be defined in terms of uniqueness conditions placed upon the composition series of the primitive ideals of the algebra. It is known that uniserial algebras can be characterized as those algebras all of whose residue class algebras are of a certain Frobenius type. The purpose of this paper is to give an extension of this result and to give similar characterizations of generalized uniserial algebras.

2. Definitions and notation. An idempotent is a nonzero element $e$ such that $e^{2}=e$. A primitive idempotent is one which cannot be written as a sum of two orthogonal idempotents. If $e$ is a primitive idempotent, then the left ideal $A e$ is called a primitive left ideal and the right ideal $e A$ is called a primitive right ideal. If a primitive left ideal $A e$ is dual to some primitive right ideal $f A$, then $A e$ and $f A$ are called dominant ideals. If $A e$ and $A f$ are primitive left ideals and if $A f$ is $A$-isomorphic, as a left $A$-module, to some subideal of $A e$, then $A f$ is said to be subordinate to $A e$. If $A e$ and $A f$ are primitive left ideals and if there exists a set $L_{1}, \cdots, L_{n}$ of subideals of $A e$ such that $A f$ is $A$-isomorphic to a subideal of the direct sum $\sum_{i=1}^{n} L_{i}$, then $A f$ is said to be weakly subordinate to $A e$. Let $A e_{1}, \cdots, A e_{m}$ be a collection of primitive ideals and let $L_{1}, \cdots, L_{n}$ be a set of ideals such that each $L_{i}$ is a subideal of some $A e_{j}$. If a primitive left ideal $A f$ is $A$-isomorphic to a subideal of $\sum_{i=1}^{n} L_{i}$ then $A f$ is said to be weakly subordinate to the set $\left\{A e_{j}\right\}_{j=1}^{m}$. Note that if $A f$ is subordinate to $A e$ then it is weakly subordinate to $A e$, and if $A f$ is weakly subordinate to $A e$ then it is weakly subordinate to any set of

Presented to the American Mathematical Society, December 29, 1955 under the title Characterizations of uniserial and generalized uniserial algebras in terms of Frobenius type algebras and on December 1, 1956 under the title $A$ note on generalized uniserial algebras; received by the editors March 25, 1957.

(1) This paper is composed partly of the results of Chapter IV of the author's dissertation written under the direction of R. M. Thrall and accepted by the University of Michigan in June, 1953. Theorems 1 and 2 were obtained in the dissertation which was done under ONR contract N8-ONR-71400. Lemma 8, Theorem 3 and the present proof of Theorem 2 were obtained after the original work. 
ideals which contain $A e$ or an ideal $A$-isomorphic to $A e$. Subordinate and weakly subordinate right ideals are defined in a similar manner.

The main classes of Frobenius type algebras are:

1. QF-3 algebras: Every primitive ideal (left or right) is either dominant or is weakly subordinate to a set of dominant ideals. (It has been proved by Thrall [8, Theorem 5] that this is equivalent to the assumption that $A$ has a unique minimal faithful representation) [8].

2. QF-3* algebras: Every primitive ideal is either dominant or is weakly subordinate to some dominant ideal.

3. QF-2 algebras: Every primitive ideal is either dominant or is subordinate to some dominant ideal. (Thrall proved [8, Theorem 1] that this is equivalent to assuming that every primitive ideal has a unique minimal subideal) [8].

4. Quasi-Frobenius algebras: Every primitive ideal is dominant $[3 ; 4]$.

5. Frobenius algebras: The algebra $A$ considered as a left $A$-module is dual to $A$ considered as a right $A$-module $[3 ; 4]$.

6. Weakly symmetric algebras: For every primitive idempotent $e$, the left ideal $A e$ is dual to the right ideal $e A$ [6].

These classes have been defined in descending order of generality. Thus, any one of the classes is properly contained in each of the classes defined before it. For proofs of these inclusions and for further properties of these classes of algebras see the references cited after each definition.

The main classes of uniserial type algebras are:

1. Generalized uniserial algebras: Every primitive ideal has only one composition series [5].

2. Uniserial algebras: Every primitive ideal has only one composition series and the algebra is the direct sum of two-sided ideals each of which is a primary algebra. (A primary algebra is one whose residue class algebra modulo the radical is simple) $[2 ; 3 ; 4]$.

It is known [2, Theorem 2; 5, Lemma 2] that each of the following gives a characterization of a uniserial algebra $A$ :

a. For every two-sided ideal $Z$ of $A, A / Z$ is a Frobenius algebra.

b. For every two-sided ideal $Z$ of $A, A / Z$ is a weakly symmetric algebra.

The main theorems of this paper give a further characterization of uniserial algebras and similar characterizations for generalized uniserial algebras. Theorem 1 states that an algebra is generalized uniserial if and only if for every two-sided ideal $Z$ of $A, A / Z$ is a QF-2 algebra. By use of Theorem 1, a further characterization of uniserial algebras is obtained. This is given by Theorem 2 which states that an algebra $A$ is uniserial if for every two-sided ideal $Z$ of $A, A / Z$ is quasi-Frobenius. This result has also been obtained by Osima [7; Theorem 12] by using basic algebras. An example will be given to show that the requirement that each $A / Z$ be QF-2 does not imply that $A$ is uniserial. Theorem 3 extends Theorem 1 , showing that it is sufficient to as- 
sume that each $A / Z$ is $Q F-3 *$ in order to imply that $A$ is a generalized uniserial algebra.

\section{Generalized uniserial algebras.}

Lemma 1. If $L$ is a primitive left ideal of $A$ and $L^{\prime}$ is a subideal of $L$ contained in every composition series of $L$, then $L^{\prime} A$ is the minimal two-sided ideal containing $L^{\prime}$ and the following equations hold: $L \cap L^{\prime} A=L^{\prime} L=L^{\prime}$.

Proof. Since $A\left(L^{\prime} A\right) A=\left(A L^{\prime}\right)(A A) C L^{\prime} A$, it follows that $L^{\prime} A$ is a twosided ideal of $A$. If $S$ is any two-sided of $A$ such that $L^{\prime} \subset S$ then $L^{\prime} A \subset S A \subset S$. Thus, $L^{\prime} A$ is the minimal two-sided ideal containing $L^{\prime}$. Since $L$ is a primitive ideal, i.e., $L=A e$, the algebra $A$, considered as a left $A$-module, can be written as a direct sum of left ideals, $A=L+M$, where $M=A(1-e)$. Thus, $L^{\prime} A$ $=L^{\prime} L \dot{+} L^{\prime} M$, where $L^{\prime} L \subset L$ and $L^{\prime} M \subset M$. Therefore, $L^{\prime} A \cap L=L^{\prime} L$.

Since $L^{\prime}$ is contained in every composition series of $L$, it is contained in every refinement of the Loewy series $L \supset N L \supset N^{2} L \supset \cdots \supset N^{s} L=0$, where $N$ is the radical of $A$. This implies that $L^{\prime}$ is one of the terms of the Loewy series $\left[1 ;\right.$ pp. 102-104]. Thus, for some integer $k, N^{k} L=L^{\prime}$ and so $L^{\prime} L$ $=\left(N^{k} L\right) L=N^{k}(L L)=N^{k} L=L^{\prime}$.

A similar lemma holds for primitive right ideals, and the proof would be analogous to that given above. (Lemmas will be stated and proved only for left ideals but will be applied if either the lemma for left ideals or the corresponding one for right ideals is needed.)

Lemma 2. If $A$ is a generalized uniserial algebra and $Z$ is a two-sided ideal of $A$, then $A / Z$ is a generalized uniserial algebra.

Proof. Consider the residue class algebra $A / Z$. Let $L^{\prime}$ be any primitive left ideal of $A / Z$. Let $L$ be the set of all elements of $A$ whose residue classes mod $Z$ are elements of $L^{\prime}$ in $A / Z$. Then $L$ is a left ideal of $A$ and a primitive ideal, $L=A e$. Since $A$ is generalized uniserial, $L$ has a unique composition series: $L=L_{m} \supset L_{m-1} \supset \cdots \supset L_{1} \supset L_{0}=0$. But $L \cap Z=Z e$ and $Z e$ is a subideal of $L$, and hence for some $n, L_{n}=Z e$. Moreover, $L / Z e=L^{\prime}$. For $i$ such that $n<i \leqq m$ define $L_{i}^{\prime}$ to be $L_{i} / Z e$. Then, each $L_{i}^{\prime}$ is a subideal of $L^{\prime}$ and the $\left\{L_{i}^{\prime}\right\}_{i=n+1}^{m}$ form a composition series for $L^{\prime}$. Let $M^{\prime}$ be any subideal of $L^{\prime}$. Let $M$ be the set of elements of $A$ whose residue classes are elements of $M^{\prime}$. Thus, $M$ is a left ideal of $A$ and is a subideal of $L$. But $M$ must be one of the $L_{i}$ since $L$ has only one composition series. However, this implies that $M^{\prime}$ is one of the $L_{i}^{\prime}$ defined above and therefore, $L^{\prime}$ has a unique composition series.

Similarly, it can be shown that any primitive right ideal of $A / Z$ has only one composition series. Thus $A / Z$ is a generalized uniserial algebra.

THeOREM 1. An algebra $A$ is a generalized uniserial algebra if and only if for every two-sided ideal $Z$ of $A$, the residue class algebra $A / Z$ is a QF-2 algebra. 
Proof. 1. Assume that $A$ is generalized uniserial. Let $Z$ be any two-sided ideal of $A$. By Lemma $2, A / Z$ is generalized uniserial and hence every primitive ideal of $A / Z$ has a unique composition series. This implies that every primitive ideal of $A / Z$ has a unique minimal subideal and thus, $A / Z$ is a QF-2 algebra.

2. Assume that for every two-sided ideal $Z$ of $A, A / Z$ is a $Q F-2$ algebra. Let $L$ be any primitive left ideal of $A$ and let $L=L_{n} \supset L_{n-1} \supset \cdots \supset L_{1} \supset L_{0}$ $=0$ be any composition series of $L$. Since $A$ is $Q F-2, L$ has a unique minimal subideal. Thus, $L_{1}$ is this unique minimal subideal and is contained in every composition series of $L$. Proceeding by induction, assume that $L_{k-1}$ is contained in every composition series of $L$. Denote $L_{k-1}$ by $L^{\prime}$ and $L^{\prime} A$ by $Z$. Since $A=L \dot{+} M$, it follows that $A / Z=L / L \cap Z \dot{+} M / M \cap Z$. Applying Lemma 1 , it follows that $L \cap Z=L^{\prime}$ and so $L / L \cap Z=L / L^{\prime}$. If $L / L^{\prime}$ is zero then $L$ has a unique composition series. Otherwise, $L / L^{\prime}$ is a primitive ideal of $A / Z$, thus $L / L^{\prime}=(A / Z) e^{\prime}$ where $e^{\prime}$ is the residue class of $e$ in $A / Z$. But since $A / Z$ is $Q F-2, L / L^{\prime}$ has a unique minimal subideal $L_{0}^{\prime}$. Let $L^{*}=\left\{x \in L \mid x+L^{\prime} \in L_{0}^{\prime}\right\}$. Then $L^{*}$ is a left ideal of $A$ and is a subideal of $L$ and moreover, $L_{k-1}$ is a maximal subideal of $L^{*}$. Thus, both $L_{0}^{\prime}$ and $L_{k} / L_{k-1}$ are minimal subideals of $L / L_{k-1}$ and thus are the same. This implies that $L^{*}=L_{k}$ and so $L_{k}$ is contained in every composition series of $L$. Thus, by induction, each $L_{n}$ is contained in every composition series of $L$ and hence, $L$ has only one composition series.

Similarly, it can be shown that each primitive right ideal has only one composition series. Thus, $A$ is a generalized uniserial algebra.

\section{Uniserial algebras.}

Lemma 3. Let $L$ be a primitive left ideal and $L^{\prime}$ a subideal contained in every composition series of $L$. If $L$ is $A$-isomorphic to some left ideal $M$ and if $M^{\prime}$ is the image of $L^{\prime}$ under the isomorphism then $L^{\prime} M=M^{\prime}, M^{\prime} \subset L^{\prime} A$ and $M^{\prime} A \subset L^{\prime} A$.

Proof. Let $\phi$ be the $A$-isomorphism from $L$ onto $M$. Thus, $\phi(L)=M$ and $\phi\left(L^{\prime}\right)=M^{\prime}$. By Lemma $1, L^{\prime} L=L^{\prime}$. Thus, $L^{\prime} M=L^{\prime} \phi(L)=\phi\left(L^{\prime} L\right)=\phi\left(L^{\prime}\right)$ $=M^{\prime}$. Thus, $M^{\prime}=L^{\prime} M \subset L^{\prime} A$ and so $M^{\prime} A \subset L^{\prime} A A=L^{\prime} A$.

Lemma 4. If $L$ is a primitive left ideal with a unique minimal subideal $L^{\prime}$ then $L^{\prime} A$ is the union of the minimal subideals of all ideals which are $A$-isomorphic to $L$.

Proof. Let $v$ be a nonzero element of $L^{\prime} A$. Then $v=y z$, where $y \in L^{\prime}$ and $z \in A$. Since $L=A e$ it follows that $L^{\prime} e=L^{\prime}$ and $y e=y$. Thus $v=(y e) z=y(e z)$. Let $w=e z$ and consider the mapping $\phi$ from $A e$ to $A w$ defined by $\phi(x)=x w$. Then $\phi$ is a homomorphism onto $A w$ and its kernel is either zero or contains $L^{\prime}$ the unique minimal subideal of $L$. If $L^{\prime}$ were in the kernel it would follow that $L^{\prime} w=0$ and this would imply that $w=0$. However, since $v$ is not zero, $w$ is not zero and so $L^{\prime}$ cannot be in the kernel of $\phi$. Thus, $\phi$ is an $A$-isomor- 
phism and $\phi\left(L^{\prime}\right)$ is the unique minimal subideal of $A w$. But $v \in L^{\prime} w=\phi\left(L^{\prime}\right)$. Thus, $v$ is an element of the minimal subideal of $A w$. Hence $L^{\prime} A$ is contained in the union of all minimal subideals of ideals which are $A$-isomorphic to $L$. Lemma 3 shows that $L^{\prime} A$ contains all such minimal ideals, and so $L^{\prime} A$ is the union of the minimal subideals of all ideals which are $A$-isomorphic to $L$.

Lemma 5. If $L$ is a primitive left ideal dual to a primitive right ideal $R$ and if $L^{\prime}$ and $R^{\prime}$ are the unique minimal subideals of $L$ and $R$ respectively, then $L^{\prime} A=A R^{\prime}$.

Proof. Since $R^{\prime}$ is the unique minimal subideal of $R$ there exists an integer $r$ such that $R^{\prime}=R N^{r}$, where $N$ is the radical of $A$. Since $R$ and $L$ are dual it follows that $L^{\prime}=N^{r} L$. Thus, $R^{\prime} L=\left(R N^{r}\right) L=R\left(N^{r} L\right)=R L^{\prime}$. Since $R^{\prime}$ is a right ideal $R^{\prime} L \subset R^{\prime}$ and since $L^{\prime}$ is a left ideal $R L^{\prime} \subset L^{\prime}$. Thus, $R^{\prime} L \subset R^{\prime} \cap L^{\prime}$. Since $L$ and $R$ are dual, if $y$ is an element of $R$ such that $y L=0$ then $y=0$. Thus, since $R^{\prime} \neq 0$ it follows that $R^{\prime} L \neq 0$ and hence, $R^{\prime} \cap L^{\prime} \neq 0$. Let $z$ be a nonzero element of $R^{\prime} \cap L^{\prime}$. Then $A z \subset A L^{\prime}=L^{\prime}$ and $A z$ is a nonzero left ideal. But since $L^{\prime}$ is minimal, this implies that $A z=L^{\prime}$. Similarly, $z A \neq 0$, $z A \subset R^{\prime} A=R^{\prime}$ and hence $z A=R^{\prime}$. Thus, $L^{\prime} A=(A z) A=A(z A)=A R^{\prime}$.

Lemma 6. If $A e$ is a primitive left ideal and $M$ is a left $A$-module with a unique maximal submodule $M_{1}$ and $M / M_{1} \cong A e / N e$ then there exists a homomorphism from Ae onto $M$.

Proof. See Nakayama [3, Lemma 1, p. 613].

Lemma 7. Let $A$ be a quasi-Frobenius algebra. Let $L$ be a primitive left ideal dual to a primitive right ideal $R$. Let $L^{\prime}$ and $R^{\prime}$ be the unique minimal subideals of $L$ and $R$ respectively. Assume $L^{\prime} \neq L$ and denote $L^{\prime} A$ by $Z$. If $A / Z$ is also quasi-Frobenius then $L / L \cap Z$ is dual to $R / R \cap Z$.

Proof. By Lemma $1, L \cap Z=L^{\prime} L=L^{\prime}$ and so, $L / L \cap Z=L / L^{\prime}$. By Lemma 5, $L^{\prime} A=A R^{\prime}$ and so, $Z=A R^{\prime}$. Thus, since Lemma 1 holds for right ideals, $R \cap Z=R R^{\prime}=R^{\prime}$ and so, $R / R \cap Z=R / R^{\prime}$. Since $L$ and $R$ are dual and $L^{\prime} \neq L$ it follows that $L / L^{\prime} \neq 0, R^{\prime} \neq R$ and $R / R^{\prime} \neq 0$. Denote $L / L^{\prime}$ by $L_{1}$ and $R / R^{\prime}$ by $R_{1}$. It can be shown that $L_{1}$ and $R_{1}$ are primitive ideals of $A / Z$. Since it is assumed that $A / Z$ is also quasi-Frobenius, $L_{1}$ must be dual to some primitive right ideal $R^{*}$ of $A / Z$. If $R^{*}$ is $A$-isomorphic to $R_{1}$ the lemma is proved since this implies that $R_{1}$ is dual to $L_{1}$. Therefore, assume that $R^{*}$ is not $A$-isomorphic to $R_{1}$. Then $R^{*}$ can be considered as an ideal of $A$ itself, since by Lemma 4 for right ideals, $Z$ is the union of all minimal subideals of all ideals of $A$ which are $A$-isomorphic to $R$. But as an ideal of $A, R^{*}$ is dual to some primitive ideal $L^{*}$ of $A$. Thus $L_{1}$ is $A$-isomorphic to $L^{*}$. Since $L / L^{\prime}=L_{1}$, and since $L$ and $L^{*}$ are both primitive ideals of $A$ and hence have unique maximal subideals, Lemma 6 can be applied to give a homomorphism from $L^{*}$ onto $L$. However, this is impossible since $L^{*}$ is isomorphic to $L / L^{\prime}$. Thus, the assumption that $R^{*}$ is not isomorphic to $R_{1}$ is false and the lemma is proved. 
Theorem 2. If for every two-sided ideal $Z$ of $A, A / Z$ is a quasi-Frobenius algebra, then $A$ is a uniserial algebra.

Proof. Since every quasi-Frobenius algebra is also a QF-2 algebra, Theorem 1 shows that $A$ is a generalized uniserial algebra. It remains to show that $A$ is the direct sum of two-sided ideals which are themselves primary algebras. By use of the Wedderburn structure theorems it is possible to choose a set of mutually orthogonal primitive idempotents whose sum is the identity element. Let $e_{i j}, i=1, \cdots, n, j=1, \cdots, f(i)$, be the set of idempotents ordered so that $A e_{i j}$ is $A$-isomorphic to $A e_{h k}$ if and only if $i=h$. It will follow that $e_{i j} A$ is $A$-isomorphic to $e_{h k} A$ if and only if $i=h$. Let $E_{i}=\sum_{j=1}^{f(1)} e_{i j}$. (For details see $[1 ; 2 ; 3]$.)

Fix an integer $i$ and the corresponding integer $h$ such that $A e_{i j}$ is dual to $e_{h k} A$. Let $L$ denote $A e_{i 1}$ and $R$ denote $e_{h 1} A$. Let $L^{\prime}$ and $R^{\prime}$ denote the unique minimal subideals of $L$ and $R$ respectively. By Lemma $1, L^{\prime} A$ is the minimal two-sided ideal containing $L^{\prime}$ and by Lemma $5, L^{\prime} A=A R^{\prime}$. Denote this twosided ideal by $Z$. It is known $[1$, Theorem $1.6 \mathrm{~B}$, p. 8$]$ that $Z$ can be written as a direct sum of left ideals and by Lemma 4 , this sum can be taken to be $\sum_{j=1}^{f(i)} L_{i j}^{\prime}$, where $L_{i j}^{\prime}$ is the unique minimal subideal of $A e_{i j}$. Thus, $Z$ is contained in $A E_{i}$, where $A E_{i}=\sum_{j=1}^{g(t)} A e_{i j}$. By a similar argument $Z$ is contained in $E_{h} A$, where $E_{h} A=\sum_{k=1}^{g(h)} e_{h k} A$. Thus, $Z \subset A E_{i} \cap E_{h} A$.

Consider the residue class algebra $A / Z$. Since $A / Z$ is quasi-Frobenius, Lemma 7 can be applied and $L / L^{\prime}$ and $R / R^{\prime}$ are dual unless they are zero. If $L / L^{\prime}$ and $R / R^{\prime}$ are zero then $Z=A E_{i}=E_{h} A$ and $Z$ is a component of $A$ written as the sum of two-sided ideals.

Construct a sequence of two-sided ideals $\left\{Z_{s}\right\}$ with the following properties: (1) for each $s, Z_{s} \subset A E_{i} \cap E_{h} A$ and (2) for each $s, Z_{s-1}$ is properly contained in $Z_{s}$. Let $Z_{1}=Z, L_{1}=L^{\prime}, R_{1}=R^{\prime}$ and $A_{1}=A / Z_{1}$. Then $L / L_{1}$ is dual to $R / R_{1}$ in $A_{1}$. Assume that $Z_{p-1}, L_{p-1}, R_{p-1}$, and $A_{p-2}$ have been defined. Then by Lemma 7 , either $Z_{p-1}=A E_{i}=E_{h} A$ or $L / L_{p-1}$ is dual to $R / R_{p-1}$ in $A / Z_{p-1}$. Let $A_{p-1}=A / Z_{p-1}$, let $L_{p}^{*}$ be the unique minimal subideal of $L / L_{p-1}$ in $A_{p-1}$, and let $Z_{p}^{*}=L_{p}^{*} A_{p-1}$. Let $Z_{p}$ be the set of all elements of $A$ whose residue classes $\bmod Z_{p-1}$ are elements of $Z_{p}^{*}$. Thus, $Z_{p}$ will be a two-sided ideal of $A$ and it follows that $Z_{p} \subset A E_{i} \cap E_{h} A$ and that $Z_{p}$ properly contains $Z_{p-1}$, by an argument similar to that used to show that $Z_{1}$ satisfied these conditions. But, since $A$ is finite dimensional, the sequence must terminate with some $Z_{q}=A E_{i}=E_{h} A$. Thus $A E_{i}=E_{h} A$ and this implies that $i=h$. Hence, $A E_{i}$ is a direct component of $A$ written as a sum of two-sided ideals. Moreover, $A E_{i} / N E_{i}$ is simple, where $N$ is the radical of $A$ and $N E_{i}$ is the radical of $A E_{i}$. Hence $A E_{i}$ is a primary algebra. Therefore, $A$ is a generalized uniserial algebra and is the sum of two-sided ideals which are themselves primary algebras. Thus, $A$ is a uniserial algebra and the theorem is proved.

Theorem 2 cannot be extended in the sense that it is not sufficient to assume that each $A / Z$ is QF-2 in order to prove that $A$ is a uniserial algebra. 
As a counter-example consider the total triangular matrix algebra $T_{n}, n>1$, of all $n$ by $n$ matrices over $K$ with zeros above the main diagonal. The algebra $T_{n}$ is generalized uniserial and thus, by Theorem 1 , each $A / Z$ is $Q F-2$. But $T_{n}$ cannot be written as a sum of two-sided ideals which are themselves primary algebras and thus $T_{n}$ is not a uniserial algebra.

\section{QF-3* algebras.}

Lemma 8. Let Ae be dual to $f A$, where e and $f$ are primitive idempotents. If, in addition to a unique minimal subideal $L_{1}$, Ae also has a unique subideal $L_{2}$ with $L_{1}$ as a maximal subideal and if $L_{2} / L_{1}$ and $L_{1}$ are $A$-isomorphic as left $A$-modules then both $A e$ and $f A$ have unique composition series and all of the constituents of the series are $A$-isomorphic.

Proof. Choose any composition series for $A e: A e=L_{n} \supset L_{n-1} \supset \cdots \supset L_{2}$ $\supset L_{1} \supset L_{0}=0$ and any composition series for $f A: f A=R_{n} \supset R_{n-1} \supset \cdots \supset R_{2} \supset R_{1}$ $\supset R_{0}=0$. Since any primitive ideal has a unique maximal subideal, the ideals $L_{n-1}$ and $R_{n-1}$ are uniquely determined. Thus, $L_{n-1}=N e$ and $R_{n-1}=f N$, where $N$ is the radical of $A$. From the assumed duality between $A e$ and $f A$, the uniqueness of $L_{n-1}$ and $R_{n-1}$ implies the uniqueness of $R_{1}$ and $L_{1}$ respectively. It is assumed in addition that $L_{2}$ is uniquely determined. By duality, this implies that $R_{n-2}$ is unique. It is also assumed that $L_{2} / L_{1}$ and $L_{1}$ are $A$-isomorphic and, by duality, this implies that $R_{n} / R_{n-1}$ and $R_{n-1} / R_{n-2}$ are $A$ isomorphic. But $R_{n}=f A$ and $R_{n-1}=f N$ and so, $R_{n-1} / R_{n-2}$ is $A$-isomorphic to $f A / f N$ and $R_{n-2}$ is the unique maximal subideal of $R_{n-1}$. Thus, the hypotheses of Lemma 6 stated for right $A$-modules are satisfied and there exists an $A$ homomorphism from $f A$ onto $R_{n-1}$. By comparing the composition lengths of $f A$ and $R_{n-1}$, it is seen that the kernel of the homomorphism must be the unique minimal subideal $R_{1}$. Thus, $f A / R_{1}$ is $A$-isomorphic to $R_{n-1}$. Denote $f A / R_{1}$ by $S$ and consider any composition series of $S: S=S_{n} \supset S_{n-1} \supset \ldots \supset S_{2}$ $\supset S_{1}=0$. The subideal $S_{n-1}$ is unique in $S$ and is $R_{n-1} / R_{1}$. Since $R_{n-2}$ is unique in $R_{n}$ it follows that $S_{n-2}$ is unique in $S$ and $S_{n-2}=R_{n-2} / R_{1}$. But, since $R_{n-1}$ and $S$ are $A$-isomorphic, the uniqueness of $S_{n-2}$ in $S$ implies the uniqueness of $R_{n-3}$ in $R_{n-1}$ and hence $R_{n-3}$ is unique in $R_{n}$. Since the composition length of $f A$ is finite, a finite number of steps shows that $f A$ has a unique composition series. Since $A e$ is dual to $f A$, this implies that $A e$ has a unique composition series.

Since $f A$ has only one composition series, so do $R_{n} / R_{1}$ and $R_{n-1}$. The composition series for $R_{n} / R_{1}$ is composed of the $\left\{R_{i} / R_{1}\right\}_{i=1}^{n}$. Since $R_{n-1}$ and $R_{n} / R_{1}$ are $A$-isomorphic, it follows that corresponding constituents are $A$ isomorphic. Thus, $\left(R_{i+1} / R_{1}\right) /\left(R_{i} / R_{1}\right) \cong R_{i} / R_{i-1}$. But, in addition,

$$
\left(R_{i+1} / R_{1}\right) /\left(R_{i} / R_{1}\right) \cong R_{i+1} / R_{i}
$$

and so, $R_{i+1} / R_{i} \cong R_{i} / R_{i-1}$. Thus, all constituents of $f A$ are $A$-isomorphic. Since $A e$ and $f A$ are dual, this implies that all of the constituents $\left\{L_{i} / L_{i-1}\right\}_{i=1}^{n}$ are $A$-isomorphic and the lemma is proved. 
If the condition that $L_{2}$ is uniquely determined is dropped from the hypothesis of Lemma 8 , but it is still assumed that for some $L_{2}, L_{2} / L_{1}$ is $A$ isomorphic to $L_{1}$, neither conclusion of the lemma need hold. Consider the algebra of all matrices of the form:

$$
\left\{\begin{array}{llll}
a_{1} & 0 & 0 & 0 \\
a_{3} & a_{1} & 0 & 0 \\
a_{5} & 0 & a_{2} & 0 \\
a_{6} & a_{5} & a_{4} & a_{2}
\end{array}\right\}
$$$$
a_{i} \in K
$$

Choose as a basis for the algebra the matrices $X_{i}$ in which $a_{i}=1$ and $a_{j}=0$ for $j \neq i$. The primitive left ideal $L$ with basis $\left\{X_{1}, X_{3}, X_{5}, X_{6}\right\}$ is dual to the primitive right ideal $R$ with basis $\left\{X_{2}, X_{4}, X_{5}, X_{6}\right\}$. The ideal $X_{6} K$ is the unique minimal subideal $L_{1}$ of $L$. If $L_{2}$ is chosen as $X_{5} K+X_{6} K$ then $L_{2} / L_{1}$ is $A$-isomorphic to $L_{1}$. However, $L_{2}$ is not unique since it could be chosen as $X_{3} K+X_{6} K$. Thus, $L$ does not have a unique composition series and not all of the constituents of $L$ are $A$-isomorphic.

If the condition that $L_{2}$ is uniquely determined is assumed but the requirement that $L_{2} / L_{1}$ be $A$-isomorphic to $L_{1}$ is dropped then neither conclusion of Lemma 8 need hold. Consider the algebra of all matrices of the form:

$$
\left\{\begin{array}{llllll}
a_{1} & 0 & 0 & 0 & 0 & 0 \\
a_{5} & a_{2} & 0 & 0 & 0 & 0 \\
a_{8} & a_{6} & a_{3} & 0 & 0 & 0 \\
a_{12} & a_{9} & 0 & a_{4} & 0 & 0 \\
a_{15} & a_{13} & a_{10} & a_{7} & a_{1} & 0 \\
a_{17} & a_{16} & a_{14} & a_{11} & a_{5} & a_{2}
\end{array}\right\} \quad a_{i} \in K .
$$

Choose as a basis for the algebra the matrices $X_{i}$ in which $a_{i}=1$ and $a_{j}=0$ for $j \neq i$. Then, the primitive left ideal $L$ with basis $\left\{X_{1}, X_{5}, X_{8}, X_{12}\right.$, $\left.X_{15}, X_{17}\right\}$ is dual to the primitive right ideal $R$ with basis $\left\{X_{2}, X_{5}, X_{11}, X_{14}\right.$, $\left.X_{16}, X_{17}\right\}$. The ideal $X_{17} K$ is the unique minimal subideal $L_{1}$ of $L$ and $X_{15} K$ $+X_{17} K$ is the unique second term $L_{2}$ in any composition series of $L$. However, $L_{2} / L_{1}$ is not $A$-isomorphic to $L_{1}$ and in addition, neither conclusion of Lemma 8 holds for this algebra.

Theorem 3. If for every two-sided ideal $Z$ of $A$, the residue class algebra $A / Z$ is a QF-3* algebra then $A$ is a generalized uniserial algebra.

Proof. If every $A / Z$ were $Q F-2$ then Theorem 1 would imply that $A$ is a generalized uniserial algebra. Thus, it suffices to consider the case in which, for some two-sided ideal $Z$, the residue class algebra $A / Z$ is QF-3* but not QF-2. There is no loss in generality in considering this residue class algebra 
to be $A$ itself. Thus, it is assumed that there is some primitive ideal of $A$ which is weakly subordinate to a dominant ideal but is not subordinate to it. Consider the case in which this is a left ideal $L^{*}$ weakly subordinate to a dominant left ideal $L$.

Choose $L^{*}$ to be maximal with respect to being weakly subordinate to $L$, i.e., choose $L^{*}$ so that there is no other primitive ideal $M$ weakly subordinate to $L$ such that $L^{*}$ is weakly subordinate to $M$. Consider the collection of all primitive left ideals which are (1) either $A$-isomorphic to or subordinate to $L$ and which are (2) ideals to which $L^{*}$ is weakly subordinate. By use of the Wedderburn structure theorems choose a subset $\left\{L_{i}\right\}_{i=1}^{r}$ of this collection which is maximal with respect to having the primitive idempotents generating the $L_{i}$ mutually orthogonal. Thus, the $L_{i}$ can be taken as components of a decomposition of $A$ into a sum of primitive left ideals. Since the set $\left\{L_{i}\right\}_{i=1}^{r}$ is chosen maximal no other component of the decomposition is in the collection.

Since each $L_{i}$ is either a dominant ideal or is subordinate to a dominant ideal, each $L_{i}$ has a unique minimal subideal $L_{i}^{\prime}$ [8, Theorem 1]. By Lemma 1 , each $L_{i}^{\prime} A$ is a two-sided ideal and by Lemma 4 , each $L_{i}^{\prime} A$ is the union of the minimal subideals of all ideals which are $A$-isomorphic to $L_{i}$. Let $Z$ be the sum of the $L_{i}^{\prime} A$. Since each $L_{i}^{\prime} A$ can be written as a direct sum [1, Theorem 1.6B ], $Z$ can be written as a direct sum. Since $A$ is $Q F-3^{*}$ and since $L^{*}$ was chosen maximal, $Z$ can be taken as the direct sum of the minimal subideals $L_{i}^{\prime}$ of the $L_{i}$. Thus, $Z=\sum_{i=1}^{r} L_{i}^{\prime}$.

Consider the residue class algebra $A / Z$ and denote it by $A^{\prime}$. Since $A$ $=M+\sum_{i=1}^{r} L_{i}$ and $Z=\sum_{i=1}^{r} L_{i}^{\prime}$, it follows that

$$
A / Z=M / M \cap Z+\sum_{i=1}^{r} L_{i} / L_{i} \cap Z=M+\sum_{i=1}^{r} L_{i} / L_{i}^{\prime} .
$$

Thus, the primitive ideals of $A^{\prime}$ are either $A$-isomorphic to some $L_{i} / L_{i}^{\prime}$ or $A$-isomorphic to some component of $M$ and hence to some primitive ideal of $A$ itself. Since $L^{*} \cap Z=0, L^{*}$ can be considered as an ideal of $A^{\prime}$ as well as an ideal of $A$. Since $L^{*}$ does not have a unique minimal subideal, it cannot be a dominant ideal of $A^{\prime}$ nor be subordinate to a dominant ideal of $A^{\prime}$. Thus, since $A^{\prime}$ is assumed to be a QF-3* algebra, $L^{*}$ must be weakly subordinate to some dominant ideal of $A^{\prime}$.

Suppose that $L^{*}$ is weakly subordinate to a primitive ideal $M^{\prime}$ of $A^{\prime}$ which is $A$-isomorphic to one of the primitive ideals of $A$. Then $M^{\prime}$ could be considered as an ideal of $A$ and hence $L^{*}$ would be weakly subordinate to $M^{\prime}$ as an ideal of $A$. However, this is impossible since this would imply that $M^{\prime}$ is $A$-isomorphic to one of the $L_{i}$.

The only possible case remaining is that $L^{*}$ is weakly subordinate to one of the $L_{i} / L_{i}^{\prime}$. If this is true then, in addition, $L^{*}$ is weakly subordinate to $L / L^{\prime}$. This implies that $L / L^{\prime}$ is a dominant ideal of $A^{\prime}$ and that $L / L^{\prime}$ has a unique minimal subideal $S^{\prime}$. Let $S_{1}$ denote $L^{\prime}$ and let $S_{2}$ be the set of all ele- 
ments of $L$ whose residue classes are in $S^{\prime}$. Then, $S_{2}$ is a left ideal of $A$ and is a subideal of $L$. Thus, $S_{1}$ and $S_{2}$ are the unique first and second terms in any composition series of $L$. Since $L^{*}$ is weakly subordinate to both $L$ and $L / L^{\prime}$, it follows that $S_{1}$ and $S_{2} / S_{1}$ are $A$-isomorphic. Thus, the hypotheses of Lemma 8 are satisfied and hence $L$ has a unique composition series and all of the constituents of the series are $A$-isomorphic. However, this implies that there can be no primitive ideals which are subordinate to or weakly subordinate to $L$ without being dominant. Hence, this case is also impossible.

Thus, it is impossible to have every residue class algebra $Q F-3^{*}$ and one of them not $Q F-2$. Therefore, if every $A / Z$ is a $Q F-3^{*}$ algebra then every $A / Z$ is a $Q F-2$ algebra and by Theorem $1, A$ is a generalized uniserial algebra.

The previously known results and Theorem 2 give the following characterizations of uniserial algebras: (a) Every residue class algebra is quasiFrobenius; (b) Every residue class algebra is Frobenius; (c) Every residue class algebra is weakly symmetric. Theorems 1 and 3 give the following characterizations of generalized uniserial algebras: (a) Every residue class algebra is QF-3*; (b) Every residue class algebra is QF-2. It seems probable that a weaker condition than that of requiring that each residue class algebra be QF-3* may suffice to imply that the algebra itself is generalized uniserial.

\section{BIBLIOGRAPHY}

1. E. Artin, C. J. Nesbitt, and R. M. Thrall, Rings with minimal condition, University of Michigan Publications in Mathematics, no. 1, 1944.

2. K. Asano, Über Verallgemeinerte Abelsche Gruppe mit Hyperkomplexem Operatorenring und ihre Anwendungen, Jap. J. Math. vol. 15 (1939) pp. 231-254.

3. T. Nakayama, On Frobeniusean algebras, I, Ann. of Math. vol. 40 (1939) pp. 611-633.

4. - - On Frobeniusean algebras, II, Ann. of Math. vol. 42 (1941) pp. 1-21.

5. - Note on uni-serial and generalized uni-serial rings, Proc. Imp. Acad. (Tokyo) vol. 16 (1940) pp. 285-289.

6. T. Nakayama and C. Nesbitt, Note on symmetric algebras, Ann. of Math. vol. 39 (1938) pp. $659-668$.

7. M. Osima, Some studies on Frobenius algebras II, Math. J. Okayama Univ. vol. 3 (1954) pp. $109-119$.

8. R. M. Thrall, Some generalizations of quasi-Frobenius algebras, Trans. Amer. Math. Soc. vol. 64 (1948) pp. 173-183.

UNIVERSITY OF MICHIGAN, ANn Arbor, Mich.

University of NoRTh Carolina,

Chapel Hill, N. C. 